\title{
Application of the Brouwer and the Kakutani fixed-point theorems to a discrete equation with a double singular structure
}

\author{
Minoru Tabata ${ }^{1 *}$ (D) and Nobuoki Eshima ${ }^{2}$
}

\section{"Correspondence:}

mnrtabata@luck.ocn.ne.jp

'Department of Mathematical

Sciences, Osaka Prefecture

University, Osaka, Japan

Full list of author information is

available at the end of the article

\section{Springer}

\begin{abstract}
Applying the method consisting of a combination of the Brouwer and the Kakutani fixed-point theorems to a discrete equation with a double singular structure, that is, to a discrete singular equation of which the denominator contains another discrete singular operator, we prove that the equation has a solution.
\end{abstract}

Keywords: Positive solution; Set-valued operator; Discrete singular equation; The Brouwer fixed-point theorem; The Kakutani fixed-point theorem

\section{Introduction}

A large number of discrete models are constructed in natural and social sciences. Many of them are expressed in terms of various kinds of discrete nonlinear equations (DNEs). It is important to study the DNEs. Several DNEs have been studied mathematically (see, e.g., [1-4] and [13]), but many DNEs have not been studied fully (see, e.g., [9, pp. 13-15]). In particular, it is very difficult to study discrete singular equations (DSEs), and there have been only a few studies on DSEs (see, e.g., [11, 12], and [16]).

Fixed-point theory can play an indispensable role in overcoming the difficulties thus encountered. Moreover, it helps its own progress to apply fixed-point theory to various DSEs. In light of the close and cooperative interaction between fixed-point theory and DSEs, we find it beneficial to broaden the application of fixed-point theory to new DSEs.

On the basis of these considerations, we are concerned with applying fixed-point theory to the existence of a solution to the following new DSE:

$$
a(x)(i)=f_{1}\left(x, f_{2}(x, a(x))\right)(i), \quad i \in D .
$$

Here $x=x(i)$ denotes an unknown function contained in $L$, where $L$ denotes the set of all real-valued functions of $i \in D$. By $D$ we denote the set of all integers $i$ such that $1 \leq i \leq N$, that is, $D:=\{1, \ldots, N\}$, where $N$ is an arbitrary integer such that $N \geq 2$. We can regard $L$ as an $N$-dimensional Euclidean space, and each element of $L$ can be regarded as a point of the Euclidean space. However, for convenience, we refer to each element of $L$ not as point but as function of $i \in D$. We define $f_{k}(u, v)=f_{k}(u, v)(i), k=1,2$, as the following operators

(c) The Author(s) 2018. This article is distributed under the terms of the Creative Commons Attribution 4.0 International License (http://creativecommons.org/licenses/by/4.0/), which permits unrestricted use, distribution, and reproduction in any medium, provided you give appropriate credit to the original author(s) and the source, provide a link to the Creative Commons license, and indicate if changes were made. 
which act on $(u, v)=(u(i), v(i)) \in L \times L$ :

$$
f_{k}(u, v)=f_{k}(u, v)(i):=c_{k} \sum_{j \in D} K(i, j)\left(\frac{u(j)+b_{k}(j)}{v(j)}\right), \quad i \in D, k=1,2
$$

where $K(i, j)$ is a known function of $(i, j) \in D \times D, b_{k}(j) \in L, k=1,2$, are given functions of $j \in D$, and $c_{k}, k=1,2$, are known constants. We define $a(x)=a(x)(i)$ as an operator that has the following form:

$$
a(x)(i):=a_{i}(x(i)), \quad i \in D
$$

where $a_{i}(r)$ is a known function of $r \in \mathbb{R}$ that satisfies the following conditions for each $i \in D$ :

$$
\begin{aligned}
& a_{i}(r) \quad \text { is a continuous function of } r \in \mathbb{R}, \\
& a_{i}(r)>0 \quad \text { for all } r>0, \\
& a_{i}(0)=0, \\
& \frac{r}{a_{i}(r)} \rightarrow+\infty \quad \text { as } r \rightarrow 0+0 .
\end{aligned}
$$

It follows from (3)-(7) and (2) with $k=2$ that $f_{2}(x, a(x))$ is a discrete singular operator acting on $x=x(i)$. Moreover, observing operator (2) with $k=1$, we see that the right-hand side of (1) is a discrete singular operator of which the denominator contains the discrete singular operator $f_{2}(x, a(x))$. Hence, we can say that (1) has a double singular structure.

A large number of DSEs with the same singular structure as (1) have been constructed in spatial economics. Hence DSE (1) is not a special one. However, fixed-point theory has not been fully applied to such DSEs. Hence, in this paper, applying fixed-point theory to DSE (1), we prove that DSE (1) has a solution. The main result of this paper is Theorem 1 that is stated in Sect. 3.

\section{Methods}

We propose a method consisting of a combination of the Brouwer and the Kakutani fixedpoint theorems. Making use of the method, we prove that DSE (1) has a positive solution. This new method is widely applicable to DSEs with double singular structure (this application is discussed in the Appendix).

In this paper we impose no condition on (3) in addition to (4)-(7). In Sect. 3, we impose conditions on $K(i, j), c_{k}, k=1,2$, and $b_{k}(j), k=1,2$, and we state and discuss Theorem 1 . In Sect. 4 we prove estimates for the discrete singular operator contained in DSE (1). In Sect. 5, making use of the Brouwer fixed-point theorem, we extend this discrete singular operator to a set-valued operator with no singularity. In Sect. 6, applying the Kakutani fixed-point theorem to this set-valued operator, we prove Theorem 1. Section 7 is the conclusion section.

In this article, we make use of no advanced knowledge of DSEs and fixed-point theory. Indeed we use the Brouwer and the Kakutani fixed-point theorems, but they are ones of the most fundamental fixed-point theorems (see, e.g., [5] and [8]). In the Appendix we make use of no advanced knowledge of spatial economics. Hence, this article can be easily 
understood even without having advanced knowledge of DSEs, fixed-point theory, and spatial economics.

\section{Results and discussion}

In what follows, throughout the paper, we assume that

$$
\begin{aligned}
& b_{1}(i)>0 \quad \text { for all } i \in D, \\
& b_{2}(i)=0 \quad \text { for all } i \in D, \\
& 0<c_{1}<c_{2}, \\
& K(i, j)>0 \quad \text { for all } i, j \in D, \\
& K(i, j)=K(j, i) \quad \text { for all } i, j \in D .
\end{aligned}
$$

Indeed condition (9) is restrictive, but conditions (8) and (10)-(12) are sufficiently general. By (3) and (9), we can rewrite DSE (1) as follows:

$$
a_{i}(x(i))=c_{1} \sum_{j \in D} K(i, j)\left(\frac{x(j)+b_{1}(j)}{g(x)(j)}\right), \quad i \in D,
$$

where

$$
\begin{aligned}
& g(x)(j):=c_{2} \sum_{k \in D} K(j, k) A_{k}(x(k)), \quad j \in D, \\
& A_{i}(r):=\frac{r}{a_{i}(r)}, \quad i \in D .
\end{aligned}
$$

Applying (7) to (15), we see that (14) is a discrete singular operator acting on $x=x(i)$. This discrete singular operator is contained in the denominator of the right-hand side of (13). Hence, even if we assume condition (9), then DSE (13) retains the double singular structure.

We define the following norm in $L$ :

$$
\|v\|:=\max _{i \in D}|v(i)| .
$$

We define the following closed subset of $L$ :

$$
L_{0+}:=\{v=v(i) \in L ; v(i) \geq 0 \text { for all } i \in D\} .
$$

We divide this subset into two disjoint subsets as follows:

$$
L_{0+}=L_{0} \cup L_{+} \text {, }
$$

where

$$
\begin{aligned}
& L_{0}:=\left\{v=v(i) \in L_{0+} ; v(i)=0 \text { for some } i \in D\right\}, \\
& L_{+}:=\left\{v=v(i) \in L_{0+} ; v(i)>0 \text { for all } i \in D\right\} .
\end{aligned}
$$


It follows from (8) and (10) that

$$
\mathbf{b}:=\left(\frac{c_{1}}{c_{2}-c_{1}}\right) \mathbf{b}_{1}>0,
$$

where

$$
\mathbf{b}_{1}:=\sum_{j \in D} b_{1}(j)
$$

Making use of (17) and (21), we define the following simplex contained in $L_{0+}$ :

$$
S:=\left\{v=v(i) \in L_{0+} ; \sum_{j \in D} v(j)=\mathbf{b}\right\}
$$

Applying (5) to (15), and recalling definition (20), we see that

$$
A_{i}(v(i)) \in L_{+}, \quad \text { for all } v=v(i) \in L_{+} .
$$

Applying this result, (10), and (11) to (14), we see that

$$
g(v)(i) \in L_{+} \quad \text { for all } v=v(i) \in L_{+} .
$$

Hence, we see that

$$
\text { the right-hand sides of (13) and (14) can be defined for all } x(i) \in L_{+} \text {. }
$$

However, recalling (6), we see easily that

$$
\text { no } x(i) \in L_{0} \text { can be substituted in (14). }
$$

Hence, we define that if $x(i) \in L_{+}$satisfies (13) for each $i \in D$, then $x(i)$ is a solution to (13). The following theorem is the main result of this paper.

\section{Theorem 1}

(i) Equation (13) has a solution $x=x(i) \in L_{+}$.

(ii) If equation (13) has a solution $x=x(i) \in L_{+}$, then

$$
x=x(i) \in S
$$

Equation (13) is a new DSE that has not been fully studied. Theorem 1(i) implies that (13) has a positive solution. It follows from Theorem 1(ii) that all positive solutions are contained in the simplex (23). Theorem 1 is proved in Sect. 6.

Theorem 1 is widely applicable to many DSEs constructed in spatial economics, since those DSEs have the same double singular structure as (13). For example, applying Theorem 1 to DSEs [6, (5.3)-(5.5)] and [6, (7.1)-(7.8), (7.14)-(7.17), (14.1)-(14.12), (15.1)(15.4), (15 A.1)-(15 A.10), (16.1)-(16.8)], we can prove that there exist positive solutions to the DSEs. This application is fully discussed in the Appendix. 
Indeed we could prove the existence of solutions to DSE [6, (5.3)-(5.5)] more easily than Theorem 1 [14, 15]. However, it is difficult to apply the method developed in [14] and [15] to (13), since this method greatly depends on spatial economic properties of the known functions contained in DSE [6, (5.3)-(5.5)]. In order to prove Theorem 1, we need the method developed in this paper.

\section{Estimates for operators}

Replacing an unknown function $x$ by $u$ and $v$ in the right-hand side of (13), we define the following operator acting on $u$ and $v$ :

$$
F_{1}(u, v)=F_{1}(u, v)(i):=c_{1} \sum_{j \in D} K(i, j)\left(\frac{u(j)+b_{1}(j)}{g(v)(j)}\right), \quad i \in D
$$

Making use of this operator, we rewrite (13) as follows:

$$
a_{i}(x(i))=F_{1}(x, x)(i), \quad i \in D \text {. }
$$

Multiplying both sides of (30) by (15) with $r=x(i)$, we obtain

$$
x(i)=F_{2}(x, x)(i), \quad i \in D,
$$

where

$$
F_{2}(u, v)=F_{2}(u, v)(i):=A_{i}(v(i)) F_{1}(u, v)(i), \quad i \in D .
$$

It follows from (24) that

$$
\text { if } x=x(i) \in L_{+}, \quad \text { then (13) is equivalent to (31). }
$$

Hence, we have only to seek a fixed point of $F_{2}(x, x)$ in $L_{+}$.

It follows from (8), (17), and (20) that

$$
u(j)+b_{1}(j) \in L_{+}, \quad \text { for all } u=u(j) \in L_{0+} .
$$

Hence, observing (29) and (32), in the same way as (26) and (27) we see easily that

$$
F_{k}(u, v), \quad k=1,2 \text {, can be defined for all }(u, v) \in L_{0+} \times L_{+},
$$

but that

$$
F_{k}(u, v), \quad k=1,2 \text {, can be defined for no }(u, v) \in L_{0+} \times L_{0} \text {. }
$$

Let us obtain estimates for $F_{k}(u, v), k=1,2$, when

$$
(u, v) \in L_{0+} \times L_{+} .
$$


Lemma 1 If (37) holds, then

$$
\begin{aligned}
& F_{k}(u, v) \in L_{+}, \quad k=1,2, \\
& \lim _{(U, V) \in L_{0+} \times L_{+},(U, V) \rightarrow(u, v)} F_{k}(U, V)=F_{k}(u, v), \quad k=1,2 .
\end{aligned}
$$

Proof Applying (4), (5), (11), (15), (24), (25), (34), and (37) to (29) and (32), we obtain (38) and (39).

The following lemma is a key lemma of this paper (see (21)).

Lemma 2 If (37) holds, then

$$
\sum_{i \in D} F_{2}(u, v)(i)=M(u)
$$

where

$$
M(u):=\left(\frac{c_{1}}{c_{2}}\right) \sum_{j \in D} u(j)+\left(1-\frac{c_{1}}{c_{2}}\right) \mathbf{b} .
$$

Proof Substituting (29) and (32) in the left-hand side of (40), we see that

$$
\sum_{i \in D} F_{2}(u, v)(i)=c_{1} \sum_{i \in D} \sum_{j \in D} A_{i}(v(i)) K(i, j)\left(\frac{u(j)+b_{1}(j)}{g(v)(j)}\right) .
$$

Exchange $\sum_{i \in D}$ and $\sum_{j \in D}$, and apply (12) to the right-hand side. Recalling definition (14) with $x=v$, we see that the right-hand side of the equality thus obtained contains both $g(v)(j)$ and $1 / g(v)(j)$, which cancel each other out. Hence, we see that

$$
\sum_{i \in D} F_{2}(u, v)(i)=\left(\frac{c_{1}}{c_{2}}\right) \sum_{j \in D}\left(u(j)+b_{1}(j)\right) .
$$

Substituting (21) and (22) in the right-hand side, we obtain (40).

Let us discuss this key lemma. Observing (14) and (29), we see that (32) is expressed in terms of the double summation. Hence, the left-hand side of (40) is expressed in terms of the triple summation with double singular structure. However, the right-hand side of (40) is expressed in terms of the single summation (41) with no singularity. By (40) we overcome the difficulty caused by the double singular structure of (13). We make use of (40) to prove Lemma 3(i). We make use of Lemma 3(i) to prove Theorem 1(i). It is difficult to prove that (29) satisfies a useful equality similar to (40). This is the reason why we define (32) instead of (29).

Recalling definition (23), we see easily that

$$
S \times\left(S \cap L_{+}\right) \subseteq L_{0_{+}} \times L_{+} .
$$

Combining this inclusion relation and (35), we see that

$$
F_{2}(u, v) \quad \text { can be defined for all }(u, v) \in S \times\left(S \cap L_{+}\right) .
$$


Let us obtain estimates for (32) when

$$
(u, v) \in S \times\left(S \cap L_{+}\right) .
$$

\section{Lemma 3}

(i) If (44) holds, then

$$
F_{2}(u, v) \in S \cap L_{+} .
$$

(ii) If (44) holds and $d_{k} \subseteq D, k=1,2$, are nonempty, then

$$
0<F_{2}(u, v)(i) \leq\left(\frac{c_{1}}{c_{2}}\right)\left(\frac{\mathbf{b}+\mathbf{b}_{1}}{\left|d_{2}\right|}\right)\left(\frac{\bar{K}}{\underline{K}}\right)\left(\frac{A_{i}(v(i))}{\underline{A}\left(d_{2}, v\right)}\right) \quad \text { for all } i \in d_{1} \text {, }
$$

where

$$
\begin{aligned}
& \underline{K}:=\min _{i, j \in D} K(i, j), \quad \bar{K}:=\max _{i, j \in D} K(i, j), \\
& \underline{A}\left(d_{2}, v\right):=\min _{i \in d_{2}} A_{i}(v(i)),
\end{aligned}
$$

and we denote the number of points of $d \subseteq D$ by

$|d|$

Proof Applying (10) and definition (23) to (41), we deduce that

$$
M(u)=\mathbf{b} \quad \text { for all } u \in S \text {. }
$$

Substituting this equality in (40), recalling definition (23), and making use of (38) with $k=2$, we obtain (45).

Let us prove (ii). It follows from (11) and (47) that

$$
0<\underline{K} \leq K(i, j) \leq \bar{K} \text { for all } i, j \in D
$$

It follows from (44) that (24) holds. Applying (10), (24), and (51) to (14), we see that each term of the right-hand side of (14) with $x=v$ is positive. Hence, replacing $\sum_{j \in D}$ and $K(i, j)$ with $\sum_{j \in d_{2}}$ and $\underline{K}$, respectively, in the right-hand side of (14) with $x=v$, we obtain

$$
g(v)(j) \geq c_{2} \underline{K} \sum_{k \in d_{2}} A_{k}(v(k))>0 \quad \text { for all } j \in D
$$

Applying (16), (24), (48), and (49) with $d=d_{2}$ to this inequality, we deduce that

$$
\left\|\frac{1}{g(v)}\right\| \leq \frac{1}{c_{2} \underline{K}\left|d_{2}\right| \underline{A}\left(d_{2}, v\right)}
$$


Making use of (10), (34), (51), and (52), we see easily that each term of the right-hand side of (29) is positive. Applying this result, (24), and (51) to (29) and (32), we obtain

$$
0 \leq F_{2}(u, v)(i) \leq c_{1} A_{i}(v(i)) \bar{K}\left\|\frac{1}{g(v)}\right\| \sum_{j \in D}\left(u(j)+b_{1}(j)\right) \quad \text { for each } i \in D .
$$

Applying (22), (23), and (53) to this inequality when $i \in d_{1} \subseteq D$, we obtain (46). Note that the right-hand side of (46) is independent of $u \in S$.

\section{Set-valued operators}

The purpose of this section is to extend the discrete operator (32), which has the double singular structure, to a set-valued operator with no singularity.

Let $v \in S \cap L_{+}$be fixed. By (43) we regard $F_{2}(u, v)$ as an operator acting on $u \in S$ for each fixed $v \in S \cap L_{+}$. Making use of (45), we see that this operator is an operator from $S$ to $S \cap L_{+}$. We refer to the operator thus defined as the partially fixed operator. We denote it by the same symbol $F_{2}(u, v)$. No confusion should arise. By (43) and (45), we define $\mathbf{F}(v)$ as a set-valued operator that maps $v \in S \cap L_{+}$to the set of all fixed points of the partially fixed operator $F_{2}(u, v)$ as follows:

\section{Definition 1}

$$
\mathbf{F}(v):=\left\{u \in S ; u=F_{2}(u, v)\right\} \quad \text { for every } v \in S \cap L_{+} .
$$

In the next section, we apply the Brouwer fixed-point theorem to the partially fixed operator $F_{2}(u, v)$ for each $v \in S \cap L_{+}$in order to prove that $\mathbf{F}(v)$ is nonempty for every $v \in S \cap L_{+}$.

Recalling (36) with $k=2$, we find it difficult to define (54) for each $v \in S \cap L_{0}$. In order to overcome such a difficulty, we define $\mathbf{F}(v)$ for each $v \in S \cap L_{0}$ as follows:

\section{Definition 2}

$$
\mathbf{F}(v):=\left\{u \in S ; D_{0}(u) \supseteq D_{+}(v)\right\} \quad \text { for every } v \in S \cap L_{0},
$$

where

$$
\begin{aligned}
& D_{0}(v):=\{i \in D ; v(i)=0\}, \\
& D_{+}(v):=\{i \in D ; v(i)>0\} .
\end{aligned}
$$

Recalling (18), we see that $\mathbf{F}(v)$ defined in (54) and (55) is a set-valued operator from $S$ to $2^{S}$.

\section{Conditions of the Kakutani fixed-point theorem}

Making use of the following lemma, we apply the Kakutani fixed-point theorem to the set-valued operator $\mathbf{F}(v)$. 


\section{Lemma 4}

(i) $S$ is a nonempty, compact, and convex subset of the $N$-dimensional Euclidean space $L$.

(ii) $\mathbf{F}(v)$ is nonempty for every $v \in S \cap L_{+}$.

(iii) $\mathbf{F}(v) \subseteq S \cap L_{+}$for all $v \in S \cap L_{+}$.

(iv) $\mathbf{F}(v)$ is nonempty for every $v \in S \cap L_{0}$.

(v) $\mathbf{F}(v) \subseteq S \cap L_{0}$ for all $v \in S \cap L_{0}$.

(vi) $\mathbf{F}(v)$ is a convex subset of $S$ for every $v \in S$.

(vii) $\mathbf{F}=\mathbf{F}(v)$ has a closed graph.

(viii) $\mathbf{F}=\mathbf{F}(v)$ has no fixed point in $S \cap L_{0}$.

Proof of Lemma 4(i)-(vi) Applying (17) and (21) to (23), we see that $S$ is a simplex contained in $L_{0+}$. Hence, we obtain (i).

Let $v \in S \cap L_{+}$be fixed. Making use of (42), (45), and (39) with $V=v$, we see that the partially fixed operator $F_{2}(u, v)$ is a continuous operator from $S$ to $S \cap L_{+} \subset S$ for each fixed $v \in S \cap L_{+}$. Hence, making use of (i) of this lemma, we apply the Brouwer fixed-point theorem to the partially fixed operator $F_{2}(u, v)$ for each fixed $v \in S \cap L_{+}$. Hence, for each $v \in S \cap L_{+}$, there exists $u_{+} \in S$ such that $u_{+}=F_{2}\left(u_{+}, v\right)$. Recalling (54), we obtain (ii).

Assume that $u \in S$ and $v \in S \cap L_{+}$satisfy that $u \in \mathbf{F}(v)$. Applying this assumption to (54) and applying (45) to the right-hand side of the equality mentioned in (54), we see that $u \in S \cap L_{+}$. Hence, we obtain (iii).

Considering definitions (19), (21), (23), (56), and (57), we see easily that

$$
D_{0}(v) \text { and } D_{+}(v) \text { are nonempty proper subsets of } D \text { for all } v \in S \cap L_{0} .
$$

Making use of this result and recalling (49), we define the following function for each $v \in S \cap L_{0}$ :

$$
\begin{aligned}
& u_{0}=u_{0}(i):=\frac{\mathbf{b}}{\left|D_{0}(v)\right|} \text { for all } i \in D_{0}(v), \\
& u_{0}=u_{0}(i):=0 \quad \text { for all } i \in D_{+}(v) .
\end{aligned}
$$

Recalling (23), (56), and (57), we see easily that $u_{0} \in S$ and $D_{0}\left(u_{0}\right)=D_{+}(v)$. Applying (55) to this result, we see that $u_{0} \in \mathbf{F}(v)$. Hence, we obtain (iv).

Assume that $u \in S$ and $v \in S \cap L_{0}$ satisfy that $u \in \mathbf{F}(v)$. Applying (58) to (55), we see that the right-hand side of the inclusion relation mentioned in (55) is nonempty. Hence, the left-hand side $D_{0}(u)$ is nonempty. Recalling definitions (19) and (56), we see that $u \in S \cap L_{0}$. Hence, we obtain (v).

Let $v \in S \cap L_{+}$be fixed. Observing (29) and (32), we see easily that the partially fixed operator $F_{2}(u, v)$ is linear with respect to $u(j)+b_{1}(j)$. Applying this result to the equality mentioned in (54), we easily obtain (vi) when $v \in S \cap L_{+}$. Assume that $v \in S \cap L_{0}$. Considering definition (56) and the inclusion relation mentioned in (55), we easily obtain (vi) when $v \in S \cap L_{0}$. 
Proof of Lemma 4(vii) Assume that $\left\{\left(u^{n}, v^{n}\right)\right\}$ is a convergent sequence such that

$$
\begin{aligned}
& \left\{\left(u^{n}, v^{n}\right)\right\} \subset S \times S, \\
& u^{n} \in \mathbf{F}\left(v^{n}\right) \quad \text { for all } n \in \mathbb{N} .
\end{aligned}
$$

Applying (i) of this lemma to this sequence, we see that

$$
\left(u^{\infty}, v^{\infty}\right) \in S \times S
$$

where

$$
\left(u^{\infty}, v^{\infty}\right):=\lim _{n \rightarrow+\infty}\left(u^{n}, v^{n}\right)
$$

Applying definition (57) to (62), we see that there exists $n_{0}>0$ such that if $n \geq n_{0}$, then

$$
\begin{aligned}
& D_{+}\left(u^{\infty}\right) \subseteq D_{+}\left(u^{n}\right), \\
& D_{+}\left(v^{\infty}\right) \subseteq D_{+}\left(v^{n}\right) .
\end{aligned}
$$

We have only to prove that

$$
u^{\infty} \in \mathbf{F}\left(v^{\infty}\right)
$$

Let us prove (65) when

$$
v^{\infty} \in S \cap L_{+} .
$$

Applying definitions (20) and (57) to (66), we see that $D_{+}\left(v^{\infty}\right)=D$. Substituting this equality in (64), we deduce that $D_{+}\left(v^{n}\right)=D$ for all $n \geq n_{0}$, that is, that $v^{n} \in L_{+}$for all $n \geq n_{0}$. Combining this result and (59), we see that

$$
v^{n} \in S \cap L_{+} \quad \text { for all } n \geq n_{0} .
$$

Applying this result, (iii) of this lemma, and (54) to (60), we deduce that if $n \geq n_{0}$, then

$$
\begin{aligned}
& u^{n}=F_{2}\left(u^{n}, v^{n}\right), \\
& \left(u^{n}, v^{n}\right) \in\left(S \cap L_{+}\right) \times\left(S \cap L_{+}\right) .
\end{aligned}
$$

Making use of (42), (61), (62), (66), and (69), we can substitute

$$
(U, V, u, v)=\left(u^{n}, v^{n}, u^{\infty}, v^{\infty}\right)
$$

in (39). Applying the equality thus obtained and (62) to (68), we deduce that

$$
u^{\infty}=F_{2}\left(u^{\infty}, v^{\infty}\right)
$$

Applying this equality, (61), and (66) to (54), we obtain (65) when (66) holds. 
Let us prove (65) when

$$
v^{\infty} \in S \cap L_{0} .
$$

Making use of (18) and (iii), (v) of this lemma, we can divide the convergent sequence (59) into two disjoint subsequences as follows:

$$
\left\{\left(u^{n}, v^{n}\right)\right\}=\left\{\left(u_{0}^{n}, v_{0}^{n}\right)\right\} \cup\left\{\left(u_{+}^{n}, v_{+}^{n}\right)\right\}
$$

where

$$
\begin{array}{ll}
\left\{\left(u_{0}^{n}, v_{0}^{n}\right)\right\} \subset\left(S \cap L_{0}\right) \times\left(S \cap L_{0}\right), & u_{0}^{n} \in \mathbf{F}\left(v_{0}^{n}\right) \text { for all } n, \\
\left\{\left(u_{+}^{n}, v_{+}^{n}\right)\right\} \subset\left(S \cap L_{+}\right) \times\left(S \cap L_{+}\right), & u_{+}^{n} \in \mathbf{F}\left(v_{+}^{n}\right) \text { for all } n .
\end{array}
$$

At least one of these subsequences is a convergent infinite sequence.

Assume that (73) is a convergent infinite sequence. Applying (55) to (73), we see that

$$
D_{0}\left(u_{0}^{n}\right) \supseteq D_{+}\left(v_{0}^{n}\right)
$$

Taking the complements of both sides of (63), we deduce that if $n \geq n_{0}$, then

$$
D_{0}\left(u^{\infty}\right) \supseteq D_{0}\left(u^{n}\right)
$$

Recalling that (73) is a subsequence of (59), we replace $\left(u^{n}, v^{n}\right)$ with $\left(u_{0}^{n}, v_{0}^{n}\right)$ in (64) and (76). Combining the inclusion relations thus obtained and (75), we see that

$$
D_{0}\left(u^{\infty}\right) \supseteq D_{+}\left(v^{\infty}\right)
$$

Applying (55), (61), and (71) to this inclusion relation, we obtain (65).

Assume that (74) is a convergent infinite sequence. Applying (54) to (74), we obtain

$$
u_{+}^{n}=F_{2}\left(u_{+}^{n}, v_{+}^{n}\right)
$$

We need to obtain (65) from (78) when (71) holds. Noting that (37) is imposed on (39), we see that $(U, V, u, v)=\left(u_{+}^{n}, v_{+}^{n}, u^{\infty}, v^{\infty}\right)$ cannot be substituted in (39). Hence, we find it difficult to perform the same calculations as done when obtaining (70) from (68). In order to overcome this difficulty, we make use of (46) instead of (39).

Making use of (71), (74), and (58) with $v=v^{\infty}$, we can substitute

$$
\left(d_{1}, d_{2}, u, v\right)=\left(D_{+}\left(v^{\infty}\right), D_{0}\left(v^{\infty}\right), u_{+}^{n}, v_{+}^{n}\right)
$$

in (44) and (46). Recall that (74) is a convergent infinite subsequence of (59). Considering definition (56) with $v=v^{\infty}$, and applying (7) and (62) to (15) with $r=v_{+}^{n}(i)$, where $i \in$ $D_{0}\left(v^{\infty}\right)$, we see that

$$
\lim _{n \rightarrow+\infty} A_{i}\left(v_{+}^{n}(i)\right)=+\infty \quad \text { for all } i \in D_{0}\left(v^{\infty}\right)
$$


Applying this result to (48) with (79), we see easily that

$$
\lim _{n \rightarrow+\infty} \underline{A}\left(D_{0}\left(v^{\infty}\right), v_{+}^{n}\right)=+\infty
$$

Considering definition (57) with $v=v^{\infty}$ and making use of (4), (5), (62), and (64), we deduce that

$$
\lim _{n \rightarrow+\infty} a_{i}\left(v_{+}^{n}(i)\right)=a_{i}\left(v^{\infty}(i)\right)>0 \quad \text { for each } i \in D_{+}\left(v^{\infty}\right) .
$$

Applying this result and (62) to (15) with $r=v_{+}^{n}(i)$, where $i \in D_{+}\left(v^{\infty}\right)$, we deduce that

$$
\lim _{n \rightarrow+\infty} A_{i}\left(v_{+}^{n}(i)\right)=\frac{v^{\infty}(i)}{a_{i}\left(v^{\infty}(i)\right)}<+\infty \quad \text { for each } i \in D_{+}\left(v^{\infty}\right) .
$$

Applying this result and (80) to (46) with (79), we see that

$$
\lim _{n \rightarrow+\infty} F_{2}\left(u_{+}^{n}, v_{+}^{n}\right)(i)=0 \quad \text { for all } i \in D_{+}\left(v^{\infty}\right) .
$$

Applying this result and (62) to (78), we see that $u^{\infty}(i)=0$ for all $i \in D_{+}\left(v^{\infty}\right)$, that is, that (77) holds. Applying (55), (61), and (71) to (77), we obtain (65). Therefore we obtain (vii).

Proof of Lemma 4(viii) Assume that there exists $v_{0} \in S \cap L_{0}$ such that $v_{0} \in \mathbf{F}\left(v_{0}\right)$. Applying this assumption to (55), we see that $D_{0}\left(v_{0}\right) \supseteq D_{+}\left(v_{0}\right)$. Applying (58) with $v=v_{0}$ to this inclusion relation, we deduce that both sides of this inclusion relation are nonempty. Recalling definitions (56) and (57), we see that this inclusion relation leads us to a contradiction. Hence, we obtain (viii).

Proof of Theorem 1 Making use of Lemma 4(i), (ii), (iv), (vi), (vii) and (18), we apply the Kakutani fixed-point theorem to $\mathbf{F}=\mathbf{F}(v)$. Hence, we see that there exists $x=x(i) \in S$ such that $x \in \mathbf{F}(x)$. Applying (18), (54), and Lemma 4(viii) to this result, we deduce that $x=x(i)$ is contained in $S \cap L_{+}$and satisfies (31). Recalling (33), we obtain (i).

Note that not (44) but (37) is imposed on (40). Assume that $x \in L_{+}$satisfies (31). Substituting (31) in the left-hand side of (40) with $u=v=x$, we see that

$$
\sum_{j \in D} x(j)=M(x)
$$

Substituting (41) with $u=x$ in the right-hand side of this equality, making use of (10), and recalling definition (23), we obtain (28).

\section{Conclusions}

Applying the method consisting of the combination of the Brouwer and the Kakutani fixed-point theorems to the discrete equation with double singular structure, we prove that the equation has a positive solution and that all positive solutions to the equation are contained in the simplex (23) (Theorem 1). 


\section{Appendix}

Spatial economics is an interdisciplinary area between economics and geography. In about 1990, Krugman began seminal research in this area. His research has since grown into one of the major branches of spatial economics, and now it is known as the New Economic Geography (NEG) (see, e.g., [6] and [7]). In 2008, Krugman was awarded the Nobel Memorial Prize in Economic Sciences for his great contribution to spatial economics [10].

Many discrete models have been constructed in the NEG. Among those models, the Krugman's core-periphery model (KCP model) is one of the most important models. In the $\mathrm{KCP}$ model, economic activities are conducted at each point of a finite set of points, and each point represents a region. The economy consists of agriculture and manufacturing, and the population consists of farmers and workers. The KCP model is described by a DSE that is referred to as the wage equation [6, pp. 61-95].

Let us apply the result of this paper to the wage equation. We assume that the finite set of points is equal to $D$ defined in Sect. 1, that is, that each integer contained in $D$ represents a region. Substitute

$$
\begin{aligned}
& x(i)=\lambda(i) w(i), \quad i \in D, \\
& a_{i}(r)=\left(\frac{r}{\lambda(i)}\right)^{\sigma}, \quad i \in D, r \geq 0, \\
& b_{1}(i)=\left(\frac{1-\mu}{\mu}\right) \phi(i), \quad i \in D, c_{1}=c_{2} \mu, \\
& K(i, j)=T(i, j)^{-(\sigma-1)}, \quad i, j \in D,
\end{aligned}
$$

in (13)-(15), where $w(i)$ denotes an unknown function contained in $L_{+}, \lambda(i), \phi(i)$, and $T(i, j)$ are known functions of $i, j \in D$, and $\sigma$ and $\mu$ are known constants. In spatial economics, $w(i)$ denotes the distribution of nominal wages, and $\sigma$ and $\mu$ denote the elasticity of substitution and the manufacturing expenditure, respectively. Moreover, $\lambda(i), \phi(i)$, and $T(i, j)$ denote the distribution of workers, the distribution of farmers, and the transportcost function, respectively. Then we obtain the following DSE, which is the wage equation $[6,(5.3)-(5.5)]$ :

$$
w(i)^{\sigma}=\sum_{j \in D} Y(w(j)) G(w ; j)^{\sigma-1} T(i, j)^{-(\sigma-1)},
$$

where

$$
\begin{aligned}
Y(w(j)) & :=\mu \lambda(j) w(j)+(1-\mu) \phi(j), \\
G(w ; j)^{\sigma-1} & :=\frac{1}{\sum_{k \in D} \lambda(k)(1 / w(k))^{\sigma-1} T(j, k)^{-(\sigma-1)}} .
\end{aligned}
$$

We assume that

$$
\begin{aligned}
& \sigma>1, \quad 0<\mu<1, \\
& \lambda(i), \phi(i) \in L_{+},
\end{aligned}
$$




$$
\begin{aligned}
& T(i, j)>0 \quad \text { for all } i, j \in D, \\
& T(i, j)=T(j, i) \quad \text { for all } i, j \in D .
\end{aligned}
$$

These assumptions are the most general conditions in spatial economics [6, pp. 46-49]. It follows from these conditions that (81)-(83) satisfy (4)-(12). Hence, applying Theorem 1(i) to the wage equation (84), we see that (84) has a positive solution $w(i) \in L_{+}$.

Performing calculations similar to those done above, we can prove that there exist positive solutions to DSEs [6, (7.1)-(7.8), (7.14)-(7.17), (14.1)-(14.12), (15.1)-(15.4), (15 A.1)(15 A.10), (16.1)-(16.8)], since these DSEs are constructed as extensions of the KCP model.

\section{Acknowledgements}

The authors would like to express their deepest gratitude to Professor Yoshitsugu Kabeya for his valuable suggestions.

\section{Funding}

Minoru Tabata and Nobuoki Eshima are supported in part by Grant-in-aid for Scientific Research of Japan (15K05005, 26330045)

\section{Availability of data and materials}

Data sharing is not applicable to this article as no datasets were generated or analyzed during the current study. Materials sharing is not applicable to this article as no materials were generated or analyzed during the current study.

\section{Competing interests}

The authors declare that there is no conflict of interests regarding the publication of this paper.

\section{Authors' contributions}

Each author equally contributed to this article, read and approved the final manuscript.

\section{Author details}

${ }^{1}$ Department of Mathematical Sciences, Osaka Prefecture University, Osaka, Japan. ${ }^{2}$ Center for Educational Outreach and Admissions, Kyoto University, Kyoto, Japan.

\section{Publisher's Note}

Springer Nature remains neutral with regard to jurisdictional claims in published maps and institutional affiliations.

Received: 3 July 2018 Accepted: 9 October 2018 Published online: 01 November 2018

\section{References}

1. Anastassiou, G.A., Kester, M.: Discrete Approximation Theory. Vol. 20. World Scientific, Singapore (2016)

2. Askhabov, S.N., Karapetyants, N.K.: Discrete equations of convolution type with monotone nonlinearity. Differ. Uravn. 25(10), 1777-1784 (1989)

3. Askhabov, S.N.: Approximate solution of nonlinear discrete equations of convolution type. J. Math. Sci. 201(5), 566-580 (2014)

4. Askhabov, S.N., Karapetyants, N.K.: Discrete equations of convolution type with a monotone nonlinearity in complex spaces. Dokl. Math. 45(1), 206-210 (1992)

5. Farmakis, I., Moskowitz, M.: Fixed Point Theorems and Their Applications. World Scientific, Singapore (2013)

6. Fujita, M., Krugman, P.R., Venables, A.: The Spatial Economy: Cities, Regions, and International Trade. MIT Press, Cambridge (2001)

7. Fujita, M., Thisse, J.-F.: Economics of Agglomeration: Cities, Industrial Location, and Globalization. Cambridge University Press, Cambridge (2013)

8. Granas, A., Dugundji, J.: Fixed Point Theory. Springer, Berlin (2013)

9. Hritonenko, N., Yatsenko, Y:: Mathematical Modeling in Economics, Ecology and the Environment. Kluwer Academic, Dordrecht (1999)

10. Krugman, P.: The Official Homepage of the Nobel Prize in Economic Sciences (2008) http://www.nobelprize.org/nobel_prizes/economic-sciences/laureates/

11. Lewis, F.L., Mertzios, B.G.: On the analysis of discrete linear time-invariant singular systems. IEEE Trans. Autom. Control 35(4), 506-511 (1990)

12. Mendez, V., Fedotov, S., Horsthemke, W.: Reaction-Transport Systems: Mesoscopic Foundations, Fronts, and Spatial Instabilities. Springer, Berlin (2010)

13. Moadab, M.H. Existence of bounded solutions to nonlinear discrete equations. Libertas Math. 9, 127-132 (1989)

14. Tabata, M., et al.: The existence and uniqueness of short-run equilibrium of the Dixit-Stiglitz-Krugman model in an urban-rural setting. IMA J. Appl. Math. 80(2), 474-493 (2015)

15. Tabata, M., Nobuoki, E.: Existence of a short-run equilibrium of the Dixit-Stiglitz-Krugman model. Discrete Dyn. Nat. Soc. 2018, Article ID 2193070 (2018)

16. Vasil'ev, A.V., Vasil'ev, V.B.: On the solvability of certain discrete equations and related estimates of discrete operators. Dokl. Math. 92(2), 585-589 (2015) 